\title{
Aspectos clínicos e epidemiológicos da tuberculose em pacientes com HIV/aids
}

\author{
Clinical and epidemiological aspects of tuberculosis in patients with HIV/aids \\ Claudio José dos Santos Júnior ${ }^{1}$ (D), Thiago José Matos Rocha² (D), Valquíria de Lima Soares² (D)
}

\begin{abstract}
RESUMO
Objetivo: Avaliar aspectos clínicos e epidemiológicos da tuberculose em indivíduos HIV+ no estado de Alagoas. Métodos: Trata-se de estudo transversal e retrospectivo que analisou as notificações de coinfecção TB-HIV no período de 2001 a 2016 no estado de Alagoas, Brasil. Para elaboração de perfil da coinfecção foram empregadas variáveis clínicas e epidemiológicas dos casos novos de TB-HIV obtidas junto à Superintendência de Vigilância em Saúde do Estado de Alagoas. Resultados: Houve ascensão do número de coinfectados no período de 2001 a $2016.0 s$ pacientes soropositivos residentes no Estado apresentaram-se como sendo a maioria do sexo masculino (74,2\%), em idade economicamente produtiva $(93,7 \%)$, com baixa escolaridade e de cor negra (92,5\%). A tuberculose pulmonar foi a forma clínica mais presente $(77,3 \%)$, seguida do subtipo extrapulmonar ganglionar periférico (7,5\%). A estratégia diagnóstica mais elucidativa para o raciocínio clínico foi o exame radiográfico do tórax ( $72,5 \%)$. Houve óbito por tuberculose em $9,5 \%$ das investigações. O percentual de cura de casos novos de tuberculose em indivíduos HIV+ no estado de Alagoas ficou abaixo da meta de $85 \%$ pactuada pela OMS. Conclusão: Os resultados evidenciam a relevância epidemiológica da tuberculose como uma comorbidade de grande impacto na saúde dos indivíduos soropositivos para o HIV+ no âmbito estadual.
\end{abstract}

Palavras-chave: Epidemiologia; Infecção oportunista relacionada ao HIV; Tuberculose; Morbidade; Alagoas.

\begin{abstract}
Objective: In this study, the objective was to evaluate the clinical and epidemiological aspects of tuberculosis in $\mathrm{HIV}+$ individuals in the State of Alagoas. Methods: This was a cross-sectional and retrospective study that analyzed the reports of TB-HIV co-infection between 2001 and 2016 in the state of Alagoas, Brazil. To develop a profile of co-infection, clinical and epidemiological variables were used for new cases of HIV-TB obtained together with the Superintendence of Health Surveillance of the State of Alagoas. Results: There was a rise in the number of co-infected patients from 2001 to 2016 . Seropositive patients living in the State were the majority male (74.2\%), of economically productive age (93.7\%), with low educational level and black (92.5\%). Pulmonary tuberculosis was the most common clinical form $(77.3 \%)$, followed by the peripheral ganglion extrapulmonary subtype $(7.5 \%)$. The most diagnostic strategy for clinical reasoning was the radiographic examination of the thorax (72.5\%). There was a TB death in $9.5 \%$ of the investigations. The percentage of cure of new cases of tuberculosis in HIV+ individuals in the state of Alagoas was below the $85 \%$ goal agreed by the WHO. Conclusion: The results evidenced the epidemiological relevance of tuberculosis as a comorbidity with a great impact on the health of seropositive individuals at the state level.
\end{abstract}

Keywords: Epidemiology; Opportunistic Infections HIV-Related; Tuberculosis; Morbidity; Alagoas.

1. Acadêmico de medicina. Universidade Estadual de Ciências da Saúde de Alagoas (UNCISAL), Maceió (AL), Brasil.

2. Professor(a). UNCISAL, Maceió (AL), Brasil.

$\triangle$ Claudio José dos Santos Júnior. Faculdade de Medicina (FAMED-UNCISAL). Rua Dr. Jorge de Lima, 113 - Trapiche da Barra. CEP: 57010-300. Maceió (AL), Brasil. claudiosantos_al@hotmail.com | Recebido: 21/03/2019 | Aprovado: 17/05/2019 


\section{INTRODUÇÃO}

La milênios a tuberculose (TB) é um problema de saúde pública. A TB é uma das 10 principais causas de morte no mundo e a maior causa de mortalidade entre as doenças infecciosas ${ }^{1}$. Em todo o globo são estimados anualmente 10 milhões de novos casos e 1,57 milhão de mortes pela doença. Entre as pessoas com infecção pelo vírus da imunodeficiência humana (HIV), uma média 900 mil novos diagnósticos e 300 mil óbitos são registrados a cada ano ${ }^{1,2}$. A maior carga da TB é suportada por comunidades que enfrentam desafios socioeconômicos e grupos que trabalham e vivem em condições de alto grau de marginalização ${ }^{3,4,5}$. O Brasil figura entre os 22 países que, juntos, concentram $80 \%$ dos casos de TB no mundo. No país, são notificados aproximadamente 67 mil casos novos e ocorrem 4,5 mil mortes anuais em decorrência de TB $^{6}$. Em pacientes soropositivos para o HIV esses mesmos indicadores são crescentes e vem se apresentando de forma desigual ${ }^{7}$. No território nacional, uma das unidades da federação com as maiores taxas de incidência de HIV/aids para no decênio 2008-2017 foi o estado de Alagoas. A UF registrou crescimento de quase $2000 \%$ no número de pessoas diagnosticadas com vírus HIV em 10 anos $^{8}$. O objetivo do presente trabalho foi estudar aspectos clínicos e epidemiológicos da tuberculose em indivíduos com HIV/aids no âmbito do estado de Alagoas. Os dados aqui abordados poderão servir de substrato para a implantação de medidas preventivas da ocorrência do agravo em questão, bem como de medidas que sirvam para mitigar o seu impacto clínico no nível individual e coletivo.

\section{MÉTODOS}

Trata-se de estudo transversal, de caráter retrospectivo, com abordagem descritiva e quantitativa. Foram incluídos todos os casos novos de diagnóstico de infecções pelo Mycobacterium tuberculosis em pacientes HIV positivos residentes no estado de Alagoas, Brasil, entre 01/01/2001 a 31/12/2016. O banco de dados empregado na pesquisa foi disponibilizado pela Superintendência de Vigilância em Saúde de Alagoas (SVE-SESAU) em setembro de 2018 através do protocolo 2251/2018, tendo sido extraído do Sistema de Agravo de Notificação. Optou-se pela abordagem até $31 / 12 / 2016$ pelo fato de este ser o ano/período cujos inquéritos epidemiológicos relativos à TB apresentavam-se totalmente finalizados/consolidados pelo setor responsável quando do início da coleta de dados desta pesquisa.

A elaboração do perfil da coinfecção Tuberculose-HIV considerou as seguintes variáveis clínicas e epidemiológicas:

\begin{tabular}{ll}
\hline $\begin{array}{l}\text { Variáveis de caracterização } \\
\text { sociodemográfica }\end{array}$ & $\begin{array}{l}\text { Variáveis de } \\
\text { caracterização clínica }\end{array}$ \\
\hline Sexo & Forma clínica \\
Faixa etária & Subtipo clínico \\
Escolaridade & Exames diagnósticos \\
Etnia & Uso de antirretroviral \\
Residência & Comorbidades \\
Populações especiais & Situação de encerra- \\
& mento \\
\hline
\end{tabular}

O cálculo da incidência de TB-HIV por ano do estudo foi realizado de acordo com metodologia proposta pelo Departamento de Vigilância Epidemiológica ${ }^{9}$ e considerou a fórmula a seguir:

$$
\text { Incidência de TB-HIV }=\frac{n^{\circ} \text { de casos novos de TB com sorologia anti-HIV positivo }}{n^{\circ} \text { total da população residente no período }} \times 10^{5}
$$

Os dados foram coletados pelos autores e agrupados em Software de processamento eletrônico, tendo os dados sido organizados considerando a data de diagnóstico constante nos registros disponibilizados pela SVE-SESAU.

A apresentação das informações foi realizada por meio de gráficos e tabelas e a discussão dos achados feita à luz da literatura especializada sobre o tema.
Por se tratar de estudo realizado com o uso de dados oriundos de fontes secundárias, não foi necessária a aprovação por Comitê de Ética em Pesquisa.

\section{RESULTADOS}

De acordo com os registros da Secretaria de Estado da Saúde de Alagoas, de 01/01/2001 
a 31/12/2016 foram diagnosticados 996 sujeitos com confirmação clínico-laboratorial de coinfecção Tuberculose-HIV.

A taxa de incidência no período foi crescente, passando de 0,6/100 mil hab. em 2001 para 4,1/100 mil hab. em 2016 (Figura 1).
O ano de maior incidência foi 2016, totalizando 139 notificações e uma taxa de incidência de 4,1 casos por 100 mil habitantes (Figura 1 ).

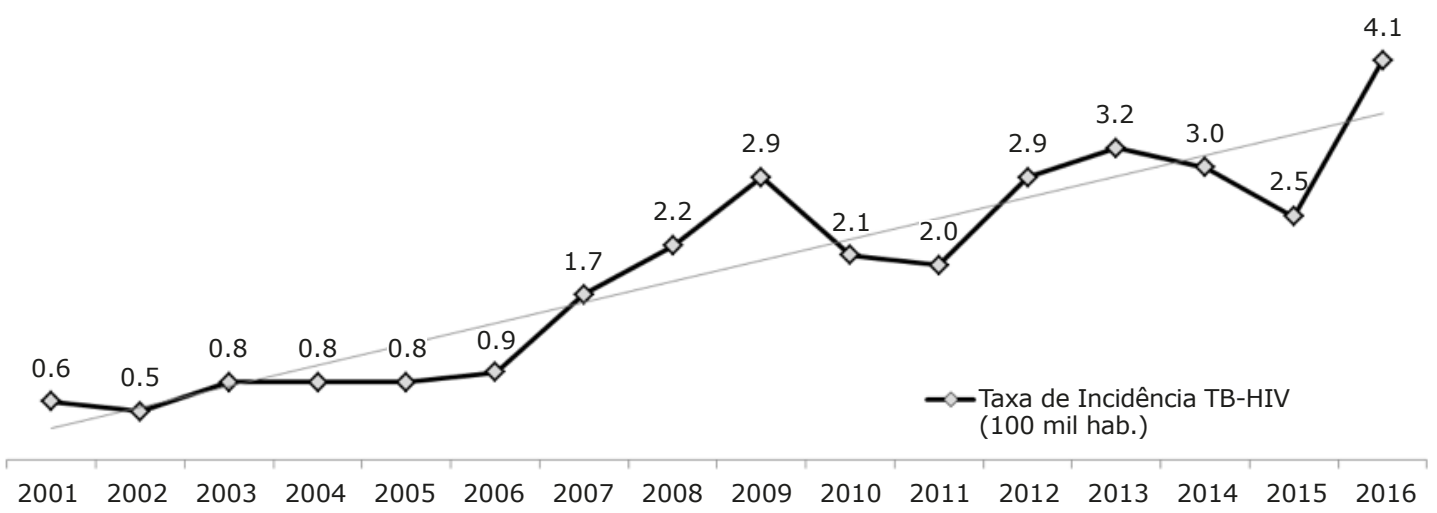

Figura 1: Taxa Anual de Incidência da coinfecção Tuberculose-HIV em Alagoas no período de 2001 a 2016

Fonte: SVE-SESAU, 2018. Elaborada pelos Autores, 2019.

Em relação às características sociodemográficas dos pacientes com diagnóstico confirmado de TB-HIV, registrou-se maior predomínio de casos entre homens, totalizando $739(74,2 \%)$ casos no sexo masculino. A distribuição de acordo com a faixa etária revelou que a maioria dos casos, $(933 ; 24,5 \%)$ situavam-se na faixa etária de 20 a 64 anos.

Quanto ao grau de formação, verificou-se que 378 (38\%) possuíam o ensino fundamental incompleto, seguido de $106(10,6 \%)$ com nenhuma escolaridade.

Classificados de acordo com a etnia, a maioria absoluta dos pacientes $(921 ; 92,5 \%)$ eram negros. Houve predomínio de indivíduos com local de moradia na zona urbana $(859 ; 86,2 \%)$.

Dos 996 acometidos, 74 (7,4\%) pertenciam a algum grupo de populações vulneráveis, sendo que o maior número de indivíduos nesta condição era de pessoas em cumprimento de medida de reclusão $(35 ; 3,5 \%)$.

A forma clínica pulmonar foi predominante, totalizando $770(77,3 \%)$ acometidos, seguida da extrapulmonar com 171 casos $(17,2 \%)$ e da forma mista com $55(5,5 \%)$ registros. Dentre os casos de TB extrapulmonar, o subtipo clínico mais comum foi a TB ganglionar com $75(7,5 \%)$ casos.

\section{Tabela 1}

Características sociodemográficas dos pacientes com HIV/aids diagnosticados com Tuberculose em Alagoas, no período de 2001 a 2016.

\begin{tabular}{lcc}
\hline Variáveis & $\mathrm{N}$ & $\%$ \\
\hline Amostra & 996 & 100 \\
\hline Sexo & 739 & 74.2 \\
Masculino & 375 & 37.7 \\
Feminino & & \\
Faixa Etária & 44 & 4.5 \\
O a 19 anos & 933 & 93.7 \\
20 a 64 anos & 18 & 1.8 \\
> 64 anos & & \\
Escolaridade & 106 & 10.6 \\
Analfabeto & 378 & 38.0 \\
EF incompleto & 61 & 6.1 \\
EF completo & 41 & 4.1 \\
EM incompleto & 55 & 5.5 \\
EM completo & 23 & 2.3 \\
ES & 332 & 33.3 \\
Ign/Branco & & \\
Etnia/Cor & 921 & 92.5 \\
Negra & 98 & 9.8 \\
Branca & 7 & 0.7 \\
Indígenas/Amarelos & 88 & 8.8 \\
Ign/Branco & \multicolumn{2}{c}{ (continua...) } \\
\hline
\end{tabular}


Tabela 1 (Continuação)

\begin{tabular}{lcc}
\hline Variáveis & $\mathrm{N}$ & $\%$ \\
\hline Residência & & \\
Urbana & 859 & 86.2 \\
Periurbana & 7 & 0.7 \\
Rural & 72 & 7.2 \\
Ign/Branco & 58 & 5.8 \\
Populações Especiais & & \\
Gestante & 4 & 0.4 \\
Situação de Rua & 12 & 1.2 \\
Privada de Liberdade & 35 & 3.5 \\
Asilos & 1 & 0.1 \\
Orfanato & 1 & 0.1 \\
Hospital Psiquiátrico & 4 & 0.4 \\
Profissional de Saúde & 4 & 0.4 \\
Outras & 13 & 1.3 \\
\hline
\end{tabular}

Fonte: SVE-SESAU, 2018. Elaborada pelos Autores, 2019.

Legenda: $\mathrm{EF}=$ Ensino Fundamental; $E M=$ Ensino Médio;

$\mathrm{ES}=$ Ensino Superior; Ign = Ignorado .

A radiografia de tórax foi o método complementar de diagnóstico mais realizado, tendo sido indicativo de TB em 722 (72,5\%). A baciloscopia de escarro foi positiva em $306(30,7 \%)$ pacientes. A cultura de secreção nasal foi realizada em $83(8,3 \%)$ casos, sendo positiva em apenas 46 $(4,6 \%)$ situações.

Quanto ao uso de terapia antirretroviral, identificou-se que em $893(89,7 \%)$ das notificações não tinham registro dessa variável e que apenas 5,9\% (59) dos pacientes diagnosticados estavam em uso da medicação para o HIV.

O hábito etílico esteve presente em 210 $(21,1 \%)$ pacientes; 59 (5.9\%) eram tabagistas; $31(3,1 \%)$ faziam uso de drogas ilícitas; $31(3,1 \%)$ possuíam diabetes; $37(3,7 \%)$ tinham diagnóstico de doença mental; e $129(13,0 \%)$ possuíam registro de outras doenças não especificadas na ficha de investigação epidemiológica.

Quanto ao desfecho dos casos, levantou-se que $444(44,6 \%)$ diagnósticos evoluíram para cura. Foi registrado abandono do tratamento em $173(17,4 \%)$ casos. Houve óbito por TB em 95 $(9,5 \%)$ investigações.
Tabela 2

Características clínicas dos casos dos casos de Tuberculose em pacientes com HIV/aids em Alagoas, no período de 2001 a 2016.

\begin{tabular}{|c|c|c|}
\hline Variáveis & $\mathrm{N}$ & $\%$ \\
\hline Amostra & 996 & 100 \\
\hline \multicolumn{3}{|l|}{ Forma clínica } \\
\hline Pulmonar & 770 & 77.3 \\
\hline Extrapulmonar & 171 & 17.2 \\
\hline Pulmonar + Extrapulmonar & 55 & 5.5 \\
\hline \multicolumn{3}{|l|}{ Subtipo clínico } \\
\hline Ganglionar Perif. & 75 & 7.5 \\
\hline Pleural & 53 & 5.3 \\
\hline Miliar & 40 & 4.0 \\
\hline Meningoencefálica & 40 & 4.0 \\
\hline Óssea & 4 & 0.4 \\
\hline Laríngea & 2 & 0.2 \\
\hline Cutânea & 1 & 0.1 \\
\hline Genitourinária & 1 & 0.1 \\
\hline Outra & 9 & 0.9 \\
\hline Ign/Branco & 171 & 17.2 \\
\hline \multicolumn{3}{|l|}{ Baciloscopia de escarro } \\
\hline Positiva & 306 & 30.7 \\
\hline Negativa & 304 & 30.5 \\
\hline Não realizada & 373 & 37.4 \\
\hline Ign/Branco & 13 & 1.3 \\
\hline \multicolumn{3}{|l|}{ Cultura de escarro } \\
\hline Positiva & 46 & 4.6 \\
\hline Negativa & 37 & 3.7 \\
\hline Não realizada & 913 & 91.7 \\
\hline \multicolumn{3}{|l|}{ Radiografia de Tórax } \\
\hline Normal & 58 & 5.8 \\
\hline Suspeito de TB & 722 & 72.5 \\
\hline Outras patologias & 9 & 0.9 \\
\hline Não realizada & 177 & 17.8 \\
\hline Ign/Branco & 30 & 3.0 \\
\hline \multicolumn{3}{|l|}{ Uso de Antirretrovirais } \\
\hline Sim & 59 & 5.9 \\
\hline Não & 44 & 4.4 \\
\hline Ign/Branco & 893 & 89.7 \\
\hline \multicolumn{3}{|l|}{ Comorbidades } \\
\hline Alcoolismo & 210 & 21.1 \\
\hline Tabagismo & 59 & 5.9 \\
\hline Diabetes & 31 & 3.1 \\
\hline Doença Mental & 37 & 3.7 \\
\hline Drogas ilícitas & 31 & 3.1 \\
\hline Outras doenças & 129 & 13.0 \\
\hline
\end{tabular}

Fonte: SVE-SESAU, 2018. Elaborada pelos Autores, 2019.

Legenda: Ign = Ignorado. 


\section{Tabela 3}

Situação de encerramento dos casos de Tuberculose em pacientes com HIV/aids em Alagoas, segundo critérios de investigação epidemiológica, no período de 2001 a 2016.

\begin{tabular}{lcc}
\hline Desfecho & $\mathrm{N}$ & $\%$ \\
\hline Cura & 444 & 44.6 \\
Abandono & 173 & 17.4 \\
Óbito por TB & 107 & 10.7 \\
Óbito por outras causas & 95 & 9.5 \\
TB resistente & 6 & 0.6 \\
Outros & 130 & 13.1 \\
Ign/Branco & 41 & 4.1 \\
Total & 996 & $100 \%$ \\
\hline
\end{tabular}

Fonte: SVE-SESAU, 2018. Elaborada pelos Autores, 2019.

Legenda: $\operatorname{Ign}=$ Ignorado; TB = Tuberculose .

\section{DISCUSSÃO}

Neste estudo, a incidência de pessoas diagnosticadas com coinfecção TB-HIV no estado de Alagoas apresentou tendência de crescimento ao longo dos anos de 2001 a 2016, indicando a ocorrência de aumento no número de pessoas com TB associada ao HIV. Corroborou-se, neste trabalho, com os achados de Gaspar et al. ${ }^{11}$ que identificaram que as taxas de incidência da tuberculose em pacientes com HIV vêm apresentando valores de detecção/diagnóstico crescentes no território nacional. No entanto, mesmo com o aumento no número de casos, durante todo o período de investigação a incidência de TB-HIV para o estado de Alagoas manteve-se inferior às taxas encontradas para o Brasil, exceto para o último ano desta pesquisa7,11.

Ao observar a presença de tuberculose em indivíduos soropositivos por sexo, percebeu-se que a presença dessa doença é duas vezes maior no sexo masculino do que no feminino. Esse achado está em conformidade com estudos realizados em outras regiões do país, como no Mato Grosso do Sul, Piauí e São Paulo, onde fora demonstrado um predomínio de $68,9 \%, 75,4 \%$ e $72,2 \%$, dos casos em homens, respectivamente ${ }^{12-14}$. A predominância de coinfecção por TB-HIV nesse grupo ainda não está bem definida, mas vem sendo atribuída ao maior autocuidado das mulheres em relação aos homens e ao estilo de vida mais cauteloso do público feminino ${ }^{15-17}$.
Houve também predomínio da coinfecção em pacientes adultos, de baixa ou nenhuma escolaridade e de cor da pele negra. Tal perfil é conclusão semelhante à de diversos outros estudos rea-

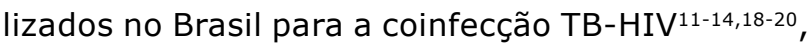
além de compatível com o perfil dos pacientes soropositivos para o HIV/aids no país ${ }^{8,21}$. O perfil é concordante também com as características da coinfecção TB-HIV nas Américas ${ }^{1,22}$. A maior taxa de coinfecção na faixa etária adulta, principalmente no grupo de adultos-jovens, vem sendo relacionada ao estilo de vida frequentemente associados à falta de consciência de sua vulnerabilidade e exposição ao vírus do HIV e à própria $\mathrm{TB}^{23}$. Nessa mesma seara, discutem-se os impactos socioeconômicos de tal associação em termos de afastamento do mercado de trabalho, diminuição da produtividade, custos previdenciários, perda de renda e gastos relacionados ${ }^{3}$. Estudos evidenciam que o desenvolvimento de TB está relacionado com as condições socioeconômicas da população e que a ocorrência da doença varia de acordo com o grupo estudado3,4,5. Aqui, em concordância com outros trabalhos, verificou-se que a cor parda e a pouca escolaridade configuram em fatores de risco para a TB e representaram determinantes sociais para a patologia, possivelmente pela maior vulnerabilidade desses segmentos.

O número de "populações especiais" acometidas pela coinfecção foi incipiente para os 16 anos de estudo, ficando em torno de $7,5 \%$ do total de casos registrados. Por sua vez, as notificações mostraram que $86,2 \%$ dos casos que receberam o diagnóstico de coinfecção do HIV com a tuberculose residiam em áreas não rurais. Estes dados estão de acordo com os achados de Pedro et al. que revelam o caráter urbano da tuberculose $\mathrm{e}$ seu predomínio em grandes cidades e ambientes de elevado fluxo de pessoas ${ }^{5}$.

Estudando as características clínicas associadas à TB, a forma pulmonar foi comprovadamente diagnosticada em 3/4 dos pacientes; a extrapulmonar, seguida da forma mista, foi responsável pela menor demanda de diagnósticos. Entre os subtipos clínicos extrapulmonares, a TB ganglionar foi a mais frequente, seguida das apresentações pleural, miliar e meningoencefálica, dados que estão de acordo com a literatura para o grau de imunocompetência na patogênese do HIV ${ }^{24,25}$. 
Quanto às estratégias diagnósticas, o método que se mostrou mais elucidativo para o raciocínio clínico foi o exame radiográfico do tórax, apresentando-se com alterações parenquimatosas sugestivas de tuberculose em 772 casos do total de 825 pacientes com diagnóstico de TB estabelecido na sua forma pulmonar e/ou mista.

Verificou-se que aproximadamente um terço dos casos analisados apresentou baciloscopia de escarro positiva, indicando TB em sua fase ativa. Esse dado está em concordância com um inquérito realizado pela Associação Latino-Americana de Tórax e European Respiratory Society que teve como finalidade verificar a aceitabilidade e a eficácia de testes diagnósticos para tuberculose na fase ativa em diversos países dos continentes americano e europeu ${ }^{27}$.

$O$ número de culturas positivas para $\circ \mathrm{M}$. tuberculosis foi proporcional à frequência de requisição desse exame. Acredita-se que a subutilização desse método se deve as difíceis condições de isolamento do agente e a própria demora na realização do procedimento. Apesar de essa metodologia ser considerada o "gold-standard" para diagnóstico e seguimento das infecções micobacterianas, seu processamento pode requerer até 60 dias para obtenção de um resultado definitivo ${ }^{26}$.

O uso de terapia antirretroviral (TARV) é reconhecidamente uma das grandes estratégias que auxiliam os pacientes soropositivos na obtenção de uma melhor qualidade de vida e na prevenção de infecções oportunistas, entre as quais se destaca a tuberculose ${ }^{28}$. Os indivíduos sororreagentes para o HIV apresentam um risco 26 vezes maior de desenvolver TB do que aqueles não infectados ${ }^{29}$. Em uma revisão sistemática com uso de meta-análise identificou-se que o uso da TARV reduz de 44 a $72 \%$ a mortalidade dos pacientes infectados pelo HIV durante o tratamento da TB ${ }^{30}$. Todavia, no presente estudo, verificou-se um total de quase $90 \%$ de "ignorados/em branco" quando do registro dessa variável no banco de dados referente aos casos de coinfecção TB-HIV em Alagoas. Tal situação evidencia que essa informação, apesar de muito relevante clinicamente, foi pouco valorizada quando da realização dos inquéritos epidemiológicos, denotando, ao mesmo tempo, uma grande limitação do processo de investigação epidemiológica do agravo pelos serviços estaduais de notificação.
Sobre a presença das comorbidades diabetes, alcoolismo, tabagismo e uso de drogas ilícitas, um estudo desenvolvido pela Sociedade Brasileira de Pneumologia identificou que a coexistência dessas condições com infecções micobacterianas pode contribuir para maus resultados no tratamento da tuberculose, evidenciando, dessa forma que os percentuais identificados para tais variáveis neste trabalho podem ter contribuído para alavancar desfechos negativos na situação de encerramento da doença no Estado ${ }^{31}$. Importante ressaltar, ademais, que o percentual de cura da tuberculose identificado para o público em estudo está muito abaixo da meta de $85 \%$ de cura de todos os casos novos de TB pactuado pela OMS .

Em concordância com a literatura especializada, verificou-se que o HIV constitui em grande desafio para o controle da TB ${ }^{11}$. Portanto, essa situação implica na necessidade de unir esforços e desenvolver ações conjuntas para o enfrentamento de ambas patologias. A articulação de ações do Programa Nacional de Controle da Tuberculose e do Programa Nacional de IST/AIDS; o estímulo a atividades com objetivo de aumentar a deteç̧ão de casos novos, aumentar a cura e diminuir o abandono de tratamento da TB; a expansão do tratamento supervisionado na Atenção Básica; e a intensificação de ações educativas em saúde, comunicação e mobilização social com enfoque na promoção e na prevenção a saúde dos indivíduos sororreagentes para o HIV se mostram como caminhos que podem ser trilhados na busca do combate à tuberculose enquanto problema da saúde no segmento em estudo ${ }^{32}$.

Importante ressaltar que, durante o estudo, verificou-se a necessidade de aperfeiçoamento do processo de preenchimento de alguns campos da ficha de notificação, de modo a melhor qualificar os esforços da vigilância da TB no Estado.

Ademais, a pesquisa seguiu, ainda, um delineamento transversal, que impossibilita definir relações de causalidade. $O$ trabalho foi restrito a população e cenário específicos de um estado, o que compromete a generalização dos achados. Outra limitação que precisa ser reconhecida é a possibilidade de subnotificação das doenças, tanto do HIV quanto da TB, sendo possível, por exemplo, que pacientes portadores de HIV e TB tenham recebido o diagnóstico de $T B$, mas não tenham sido submetidos à sorologia anti-HIV. 
Apesar dessas considerações, os resultados aqui apresentados cumprem o papel de traçar um panorama dos aspectos clínicos e epidemiológicos da tuberculose nos indivíduos HIV+ na unidade federada em análise, visto que se fundamentaram em banco de dados oficial fornecido pelo órgão de saúde estadual. A análise aqui realizada poderá servir de substrato para a implantação de medidas preventivas relacionadas à ocorrência da tuberculose em pacientes soropositivos em Alagoas, bem como de medidas que sirvam para mitigar o seu impacto clínico ao nível individual e coletivo dessa patologia.

Para futuros trabalhos, seria interessante conhecer o percentual de oferta e de cobertura do teste rápido para HIV às pessoas com diagnóstico de TB nos municípios do estado, correlacionando esses indicadores com o monitoramento dos agravos.

\section{CONCLUSÃO}

Os resultados evidenciam que houve ascensão do número de diagnóstico de Tuberculose na população sororreagente para HIV em Alagoas de 2001 a 2016. Os pacientes com coinfecção TB-HIV no Estado apresentaram-se como sendo a maioria do sexo masculino, em idade economicamente produtiva, com baixa escolaridade e de cor negra. A tuberculose pulmonar foi a forma clínica mais presente e o subtipo ganglionar periférico predominou na categorização dos casos de TB extrapulmonar. A estratégia diagnóstica mais elucidativa para o raciocínio clínico foi o exame radiográfico do tórax. O percentual de cura de casos novos de tuberculose em indivíduos HIV+ no estado de Alagoas ficou abaixo da meta de $85 \%$ pactuada pela OMS.

\section{Conflito de Interesses}

Não há.

\section{Fonte de fomento}

Não há.

\section{Aprovação do comitê de ética em pesquisa}

Não se aplica.

\section{Registro dos ensaios clínicos em base de acesso público}

Não se aplica.

\section{REFERÊNCIAS}

1. World Health Organization. Global tuberculosis report 2018. Geneva: WHO; 2018. http://www.who.int/tb/publications/global_report/en/.

2. World Health Organization. Latent TB infection: updated and consolidated guidelines for programmatic management. Geneva: WHO; 2018. http://www.who.int/tb/publications/2018/latent-tuberculosis-infection/en/.

3. Sales CMM, Nunes GF, Rogério W, Castro T, Santos BR, Maciel ELN. Tuberculosis and social issues: a systematic review of Brazilian studies. Rev Bras Pesq Saúde. 2015;17(4):156-75.

4. San Pedro A, Oliveira RM. Tuberculosis and socioeconomic indicators: systematic review of the literature. Rev Panam Salud Publica [internet]. 2013 [acesso em 15 ago 2019];33(4):294-301. doi: 10.1590/s102049892013000400009

5. Pedro AS, Gibson G, Santos JPC, Toledo LM, Sabroza PC, Oliveira RM. Tuberculosis as a marker of inequities in the context of socio-spatial transformation. Rev Saúde Pública [internet]. 2017 [acesso em 15 ago 2019];51:1-10. doi: http://dx.doi.org/10.1590/s1518-8787.2017051006533

6. Ministério da Saúde (BR). Secretaria de Vigilância em Saúde. Departamento de Doenças Transmissíveis. Panorama da tuberculose no Brasil: indicadores epidemiológicos e operacionais. Brasília (DF): Ministério da Saúde; 2014.

$7 . \quad$ Boletim Epidemiológico Tuberculose. Brasília (DF): Ministério da Saúde; 2016.

8. Boletim Epidemiológico HIV/aids. Brasília (DF): Ministério da Saúde; 2017.

9. Manual de recomendações para o controle da tuberculose no Brasil. Brasília: Ministério da Saúde; 2011.

10. . Saúde Brasil 2013: uma análise da situação de saúde e das doenças transmissíveis relacionadas à pobreza. Brasília (DF): Ministério da Saúde; 2014.

11. Gaspar RS, Nunes N, Nunes M, Rodrigues VP. Temporal analysis of reported cases of tuberculosis and of tuberculosis-HIV co-infection in Brazil between 2002 and 2012. J Bras Pneumol [internet]. 2016 [acesso em 15 ago 2019];42(6):416-22. doi: http://dx.doi.org/10.1590/ s1806-37562016000000054 
12. Baldan SS, Ferraudo AS, Andrade M. Clinical and epidemiological characteristics of tuberculosis and HIV coinfection and the association with the Human Development Index in Mato Grosso do Sul State, Brazil. Rev Pan-Amaz Saude [internet]. 2017 [acesso em 15 ago 2019];8(3):59-67. doi: 10.5123/S217662232017000300007

13. Oliveira LBO, Costar CRB, Queiroz AAFLN, Araújo TME, Sousa KAAS, Reis RK. Epidemiological analysis of tuberculosis/hiv coinfection. Cogitare Enferm [internet]. 2018 [acesso em 15 ago 2019];23(1):e51016. doi: http://dx. doi.org/10.5380/ce.v23i1.51016

14. Castrighini CC, Reis RK, Neves LAS, Galvão MTG, Gir E. Prevalence and epidemiological aspects of HIV/tuberculosis coinfection. Uerj Nurs. J [internet]. 2017 [acesso em 16 ago 2019];25:e17432. doi: http://dx.doi. org/10.12957/reuerj.2017.17432

15. Pinheiro RS, Viacava F, Travassos C, Britto AS. Gender, morbidity, access and utilization of health services in Brazil. Ciênc Saúde Coletiva [internet]. 2002 [acesso em 16 ago 2019];7(4):687-707. doi: http://dx.doi. org/10.1590/S1413-81232002000400007

16. Alcântara CCS, Kritski AL, Ferreira VG, Façanha MC, Pontes RS, Mota RS, et al. Factors associated with pulmonary tuberculosis among patients seeking medical attention at referral clinics for tuberculosis. J Bras Pneumol [internet]. 2012 [acesso em 16 ago 2019];38(5):622-9. doi: http://dx.doi.org/10.1590/S1806-37132012000500012

17. Santos JN, Sales CMM, Prado TN, Maciel EL. Factors associated with cure when treating tuberculosis in the state of Rio de Janeiro, Brazil, 2011-2014. Epidemiol Serv Saúde [internet]. 2018 [acesso em 16 ago 2019];27(3):e2017464. doi: http://dx.doi.org/10.5123/ s1679-49742018000300015

18. Barbosa IR, Costa ICC. Epidemiological study of tuberculosis-HIV co-infection in northeastern Brazil. Rev Patol Trop [internet]. 2014 [acesso em 16 ago 2019];43(1):27-38. doi: https://doi.org/10.5216/rpt. v43i1.29369

19. Ministério da Saúde (BR). Secretaria de Vigilância em Saúde. Departamento de Vigilância Epidemiológica. Coinfecção TB-HIV no Brasil: panorama epidemiológico e atividades colaborativas. Brasília: Ministério da Saúde; 2017.

20. Menezes AMF, Almeida KT, Nascimento AKA, Dias GCM, Nascimento JC. Epidemiological profile of seropositive individuals for HIV/aids. Rev enf UFPE on line [internet]. 2018 [acesso em 16 ago 2019];12(5):1225-32. doi: https://doi. org/10.5205/1981-8963-v12i5a230907p1225-1232-2018

21. Miranda LO, Araujo GBF, Andrade DFR, Carvalho ML, Silva SMF, Moreira AM, et al. Aspectos epidemiológicos da coinfecção Tuberculose/HIV no Brasil: revisão integrativa. Rev Pre Infec e Saúde [internet].2017 [acesso em 16 ago 2019];3(3):59-70. doi: https://doi.org/10.26694/ repis.v3i3.6450
22. Organização Pan-americana de Saúde. Tuberculosis in the Américas: Epidemiology, Control, and Financing; [sl.]: OMS; 2015.

23. Prado TN, Miranda AE, Souza FM, Dias ES, Sousa LK, Arakaki-Sanchez $D$, et al. Factors associated with tuberculosis by HIV status in the Brazilian national surveillance system: a cross sectional study. BMC Infect Dis [internet]. 2014 [acesso em 16 ago 2019];14:415. doi: 10.1186/1471-2334-14-415

24. Ossalé Abacka KB, Koné A, Akoli Ekoya O, Bopaka RG, Lankoandé Siri $\mathrm{H}$, Horo K. Extrapulmonary tuberculosis versus pulmonary tuberculosis: epidemiological, diagnosis and evolutive aspects. Ver Pneumol Clin [internet]. 2018 [acesso em 16 ago 2019];74(6):452-7. doi: 10.1016/j.pneumo.2018.09.008

25. Teixeira F, Raboni SM, Ribeiro CE, França JC, Broska AC, Souza NL. Human Immunodeficiency Virus and Tuberculosis Coinfection in a Tertiary Hospital in Southern Brazil: Clinical Profile and Outcomes. Microbiol Insights [internet]. 2018 [acesso em 16 ago 2019];11(1):1-8. doi: https://doi.org/10.1177/1178636118813367.

26. Amicosante $M, D^{\prime}$ Ambrosio $L$, Munoz M, Mello FCQ, Tebruegge $M$, et al. Current use and acceptability of novel diagnostic tests for active tuberculosis: a worldwide survey. J Bras Pneumol [internet]. 2017 [acesso em 16 ago 2019];43(5):380-92. doi: http://dx.doi.org/10.1590/ s1806-37562017000000219

27. Bento J, Silva AS, Rodrigues F, Duarte R. Diagnostic tools in tuberculosis. Acta Med Port. 2011;24(1):145-54.

28. Coelho LE, Escada ROS, Barbosa HPP, Santos VGV, Grinsztejn BGJ. O tratamento da coinfecção HIV-TB. Braz. J infect dis. 2016;2(5):134-48.

29. World Health Organization. TBHIV factsheet 2015. HIV-Associated Tuberculosis. Geneva: WHO; 2015. https:// bit.ly/2U7j5Ft.

30. Odone A, Amadasi S, White RG, Cohen T, Grant AD, Houben RM. The impact of antiretroviral therapy on mortality in HIV positive people during tuberculosis treatment: a systematic review and meta-analysis. PLoS ONE [internet]. 2014 [acesso em 16 ago 2019]; 9:e112017. doi: https://doi.org/10.1371 / journal.pone.0112017

31. Silva DR, Mu-oz-Torrico M, Duarte R, Galvão T, Bonini $E H$, Arbex FF, et al. Risk factors for tuberculosis: diabetes, smoking, alcohol use, and the use of other drugs. J Bras Pneumol [internet]. 2018 [acesso em 16 ago 2019];44(2):145-52. doi: https://doi.org/10.1590/ s1806-37562017000000443.

32. Ministério da Saúde (BR). Secretaria de Vigilância em Saúde. Departamento de Doenças Transmissíveis. Programa Nacional de Controle da Tuberculose. Brasília: Ministério da Saúde; 2016. 